\title{
Reflexiones sobre los retos feministas ante la globalización
}

\author{
Rosalva Aída Hernández Castillo
}

MARGARITE WALLER Y SILVIA MARCOS (eds.), 2005

\section{Dialogue and Difference: Feminisms}

\section{Challenge Globalization}

Palgrave Macmillan (serie Estudios Feministas Comparativos),

Nueva York.

E n esta época de globalización caracterizada por la fragmentación del proceso productivo de las luchas políticas y de los imaginarios colectivos, la construcción de alianzas y articulaciones entre las luchas y la elaboración de cadenas de equivalencias entre micro políticas son los retos que enfrentan en diversas regiones del planeta los sectores críticos de la academia feminista. En este esfuerzo por construir articulaciones resulta prioritario establecer diálogos y compartir experiencias entre quienes desarrollamos nuestras luchas en el llamado Tercer Mundo, o lo que algunos autores han llamado "El mundo de las dos terceras partes" (Two-Thirds Worlds ${ }^{1}$ ). Dialogue and Difference: Feminisms Challenge

${ }^{1}$ Véase Gustavo Esteva y Madhu Suri Prakash, 1998, Grassroots Post-Modernism: Remaking the Soil of Cultures, Zed Press, Londres.

\section{Reflections on the Feminist Challenges Facing Globalization}

Rosalva AÍdA HERNÁNDEZ CASTILLO: Centro de Investigaciones y Estudios Superiores en Antropología Social-Distrito Federal, México.

aidaher2005@yahoo.com.mx

Desacatos, núm. 26, enero-abril 2008, pp. 178-183.

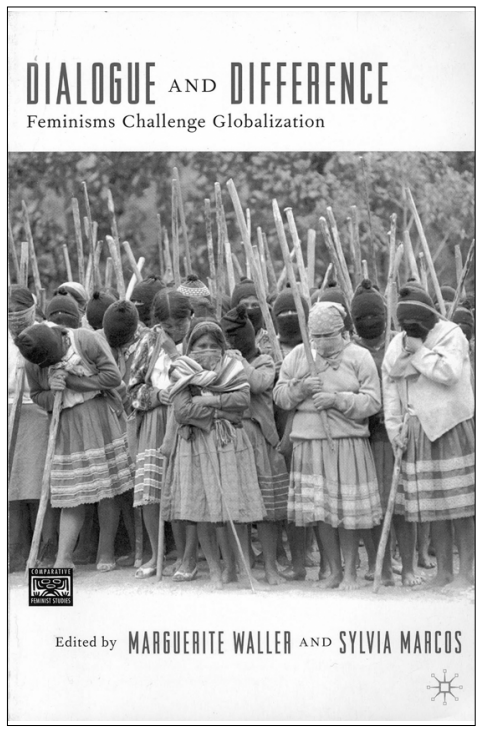

Globalization ${ }^{2}$ responde a esta necesidad reuniendo las voces y reflexiones de intelectuales feministas de México, Nigeria, Túnez, China y de la diáspora

${ }^{2}$ Una traducción al español con el título Diálogo y diferencia: retos feministas a la globalización será publicada próximamente por el Centro de Investigaciones Interdisciplinarias en Ciencias y Humanidades (CEIICH) de la Universidad Nacional Autónoma de México (UNAM). 
latinoamericana en Estados Unidos, quienes tuvieron la oportunidad de trabajar juntas en una estancia de investigación en la Universidad de Irving en California y construir un marco teórico y político compartido que le da coherencia a este libro.

Desafortunadamente, la información y las personas no fluyen tan fácilmente de sur a sur como lo hace el capital y la fuerza laboral (cuando se le requiere) de norte a sur y de sur a norte, respectivamente. El intercambio político e intelectual entre América Latina, África y Asia se ha visto limitado por barreras lingüísticas y por la precariedad de nuestras industrias editoriales e instituciones científicas y educativas. Son estos espacios privilegiados de encuentro que se dan a veces dentro de las academias del primer mundo, como el Seminario de Irving, los que nos permiten intercambiar experiencias y construir una reflexión crítica comparativa. En esta experiencia el pensamiento crítico feminista mexicano estuvo representado por Sylvia Marcos, una intelectual que se ha caracterizado por su sensibilidad hacia la diferencia cultural y sus esfuerzos por construir diálogos entre fronteras. Como editora y como autora de esta colección, Sylvia Marcos es, una vez más, puente entre tradiciones intelectuales y académicas y entre genealogías políticas distintas. En el pasado, su trabajo periodístico y académico dio a conocer en México las luchas de las Mujeres de negro y las experiencias de las mujeres bereberes, construyendo así puentes entre Latinoamérica, África y Medio Oriente. Ha tendido también puentes entre las organizaciones feministas y las organizaciones indígenas, y ahora establece uno entre los debates poscoloniales sobre la diferencia y el diálogo entre los feminismos.

El libro aborda dos grandes retos que enfrenta el feminismo hoy en día: el reconocimiento de nuestras diversidades culturales y la búsqueda de estrategias de lucha compartidas que partan de este reconocimiento. Por un lado, es cada vez más apremiante para cualquier proyecto feminista reconocer la diversidad de experiencias que marcan las identidades femeninas en el mundo y cómo las desigualdades étnicas y raciales, de clase y de preferencia sexual, determinan las maneras específicas en que construimos nuestras identidades de género. Es primordial la necesidad de reconocer cómo estas diferencias determinan la manera en que vivimos las exclusiones de género, la forma en que las conceptualizamos y las estrategias de resistencia que desarrollamos para confrontarlas. Este reconocimiento conlleva una crítica a cualquier agenda feminista universalizante. Por otro lado, es necesario dar cuenta del contexto de globalización neoliberal y opresión que marca la vida de todas las mujeres del mundo. Cómo reconocer las diferencias y hacer de ellas fortalezas para luchar contra la globalización desde arriba es el tema principal de este excelente libro.

Estos debates resultan especialmente pertinentes para el contexto mexicano en este momento histórico en el que el reconocimiento de la multicuturalidad nacional ha puesto en el centro del debate el tema de las diferencias identitarias. La tensión entre el reconocimiento de las diferencias en- tre las mujeres y la posibilidad de alianzas políticas ha sido una preocupación del feminismo mexicano, sobre todo a partir de 1994, con la salida a la luz pública del Ejército Zapatista de Liberación Nacional (EZLN) y su Ley Revolucionaria de las Mujeres.

Como nos muestra Sylvia Marcos en su capítulo de esta colección, es a partir de esa fecha que se empezó a construir a nivel nacional en México un movimiento de mujeres indígenas de diversas regiones del país, que empezaron a levantar sus voces no sólo para apoyar las demandas de sus compañeros o para representar los intereses de sus comunidades, sino para exigir el respeto a sus derechos específicos como mujeres.

$\mathrm{Al}$ mismo tiempo que las voces de las mujeres indígenas han tenido una influencia en la reconceptualización de los feminismos mexicanos, también han desempeñado un papel importante confrontando el uso de los discursos sobre los derechos de las mujeres como argumento para negar los derechos culturales de los pueblos indígenas.

Las mujeres indígenas han reivindicado el derecho a la autodeterminación y a la cultura propia, a la vez que han luchado al interior del movimiento indígena por redefinir los términos en que se entiende la tradición y la costumbre y por participar activamente en la construcción de los proyectos autonómicos.

Se trata de una reivindicación del carácter multicultural de la nación a partir de una definición más amplia de cultura, que incluye no sólo las voces y representaciones hegemónicas de 
la misma, sino la diversidad de voces y procesos contradictorios que dan sentido a la vida de un colectivo humano. Estos debates, que han ocupado un espacio especial de reflexión dentro de la academia feminista en México, pueden encontrar algunas respuestas en las experiencias y reflexiones sobre los feminismos de la diversidad que se construyen en otras regiones del mundo y que se comparten en este libro. Estos retos son abordados por las autoras de Dialogue and Difference: Feminisms Challenge Globalization en tres secciones: 1) "Encuentros", 2) "Diálogos", 3) "Reconceptualizar los derechos".

\section{Encuentros (¿o desencuentros?)}

En la primera parte, dos feministas Estados Unidos, reconstruyen los encuentros de distintas tradiciones académicas feministas con el feminismo de Estados Unidos. En su capítulo titulado "Toward an Ethics of Transnational Encounters, or 'When' Does a 'Chinese' Women Become a 'Feminist" ("Hacia una ética del encuentro transnacional: cuando una mujer china se convierte en feminista"), Shumei-Shi analiza la vida de una feminista china pionera en los estudios de las mujeres: Li Xiaojiang. Reflexiona sobre los encuentros transnacionales entre feminismos y plantea que éstos no deben ser conflictivos ni asimilacionistas, y propone que para traducir nuestros feminismos necesitamos reconocer y examinar nuestras diferencias en el momento mismo del encuentro. Para ella, una política de la

parcialidad por sobre el universalismo, la contextualización, la historización y la perspectiva crítica del conocimiento proveniente de una localidad específica son lo que permitirá un encuentro ético entre conocimientos.

Las intelectuales que cruzan fronteras deben utilizar sus múltiples posiciones de sujeto para desestabilizar los conocimientos hegemónicos.

Dando continuidad a las reflexiones de Shu-mei-Shi sobre el feminismo chino, Yenna Wu, en su capítulo "Making Sense in Chinese Feminism/ Women's Studies" ("Tener sentido dentro del feminismo chino/estudios de la mujer"), nos habla de la misma feminista pionera china, Li Xiaojiang, y de sus desencuentros con las chinasestadounidenses de la diáspora como profesora invitada en Harvard. Señala que conceptos básicos para el feminismo occidental como liberación, igualdad o lo personal son políticos. Tienen otro sentido en una sociedad comunista con una economía controlada por el Estado y una ideología que trata cada aspecto de la vida social como político.

El tema de las tensiones entre los feminismos occidentales y las construcciones locales de agendas feministas que parten de contextos culturales y concepciones distintas del sujeto es abordado también por la feminista nigeriana Obioma Nnaemeka en su capítulo "Internacional Conferences as Sites for Tansnacional Feminist Struggles: The Case of the First International Conference on Women in Africa and the African Diaspora" ("Las conferencias internacionales como lugar de luchas feministas transnacionales: el caso de la Primera Conferencia Internacional de Mujeres en África y la diáspora africana"), en el que emprende una etnografía de un encuentro entre feministas africanas y afrodescendientes estadounidenses realizado en Nsukka, Nigeria, en 1993. Esta crónica nos habla de los desencuentros entre ambos grupos: las estadounidenses cerradas a la participación de blancas y hombres son vistas como excluyentes por las africanas, que no entienden la política de identidades de Estados Unidos. Obioma trata de ser un puente entre ambas tradiciones académicas y contextualizar sus desencuentros.

En la siguiente sección, Sylvia Marcos, Margarite Waller, Shu-mei Shih y Ombioma Nnaemeka establecen diferentes diálogos y conversaciones, creando espacios para las voces de mujeres indígenas y africanas que nos remiten a epistemologías alternativas y distintas maneras de entender la lucha por la equidad y la justicia para las mujeres.

En su capítulo "The Borders Within: The Indigenous Women's Movement and Feminism in Mexico" ("Las fronteras internas: el movimiento de mujeres indígenas y el feminismo en México"), Sylvia Marcos es una vez más cronista del movimiento de mujeres indígenas, haciendo llegar al espacio internacional sus voces y experiencias ${ }^{3}$. Como estudiosa y actora

\footnotetext{
${ }^{3}$ Una versión en español previa de este capítulo ha sido publicada bajo el título de "Identidades en transformación: las prácticas feministas en el Movimiento de Mujeres Indígenas", en Paloma Bonfil y Elvia Rosa Martínez Medrano (coords.), Diagnóstico
} 
social de muchos de los procesos que describe, la autora hace una reconstrucción crítica de la historia del feminismo mexicano analizando los desencuentros entre el feminismo y el movimiento de mujeres indígenas. Pasa después a reflexionar sobre las epistemologías y cosmovisiones alternativas que dan sentido a las movilizaciones de mujeres indígenas. Ubica conceptos básicos para ellas como el de paridad en vez de igualdad, conceptualizado como "caminar parejo". Se trata de una cosmovisión que enfatiza el equilibrio entre todos los seres de la naturaleza y la visión de la Madre Tierra no sólo como propiedad de la tierra, sino como algo sagrado que se debe cuidar. Analiza también la metáfora "pensar con el corazón", utilizada en general por las mujeres indígenas como un concepto que tiene sus raíces en las culturas mesoamericanas y que va más allá de los esencialismos que vinculan a las emociones con lo femenino. Con ejemplos etnográficos, testimonios y análisis semiótico, Sylvia Marcos nos recuerda que existen distintas maneras de conocer y plantear las estrategias de lucha desde las mujeres.

De manera indirecta, Margarite Waller dialoga con Sylvia Marcos y nos muestra el efecto que estas propuestas alternativas están teniendo en los llamados feminismos del Primer Mundo. En su capítulo "One Voice Kills Both Our Voices: First World Feminism and Transcultural Feminist Engagement"

de la discriminación hacia las mujeres indígenas, Comisión Nacional para el Desarrollo de los Pueblos Indígenas, México, 2003.
("Una voz mata nuestras voces: el feminismo de Primer Mundo y los enlaces del feminismo transcultural"), la coeditora del libro analiza las desestabilizaciones del feminismo occidental al encontrarse con otras epistemologías y cosmovisiones. Poniendo en duda las propuestas posestructuralistas que siguen centradas en marcos teóricos occidentales y que muchas veces conllevan la relativización de las certezas políticas y la desmovilización, Waller señala que es más fácil deconstruirse a sí mismo (como lo hace el feminismo posmoderno) que dialogar realmente con otras epistemologías y enriquecerse con ellas.

Esta segunda sección se cierra con una conversación entre Shu-mei, Sylvia Marcos y Obioma Nnaemeka, quienes, retomando un formato innovador, hacen al lector testigo de sus propios diálogos y debates. En "Conversation on Feminist Imperialism and the Politics of Difference" ("Conversaciones sobre imperialismo feminista y las políticas de la diferencia"), las autoras realizan una autocrítica del papel que las intelectuales desempeñamos como intermediarias entre las agendas de género eurocentristas y las mujeres pobres. Hablando desde sus propias experiencias con la agenda de salud reproductiva, cuestionan cómo las definiciones amplias de ésta han sido sustituidas por perspectivas fatalistas.

Este capítulo es tal vez uno de los más sugerentes del libro, por su forma y contenido, al atreverse a ubicar al feminismo como un discurso de poder, no cuestionándolo desde afuera, sino a partir de una autocrítica a nuestro posicionamiento estructural como feministas académicas. Las autoras nos hablan de la complicidad que organizaciones e intelectuales feministas han tenido con los Estados-nación en lo que yo llamaría un doble proceso de culturización de las desigualdades de género (es decir, al presentarlas como producto de las "culturas subdesarrolladas") y desculturización de los discursos de derechos de las mujeres (al presentarlos como un discurso universal, no situado cultural ni históricamente). El discurso desarrollista y modernizador ha tendido a culpar a las "culturas indígenas" y a las "costumbres y tradiciones" de las exclusiones de las mujeres, presentando la panacea del desarrollo y los derechos de las mujeres como alternativas desculturizadas ante la exclusión.

Los derechos de las mujeres como "globalismos localizados" han sido promovidos por los Estados nacionales como parte de las iniciativas para incorporar a las mujeres en el desarrollo y cumplir con los compromisos internacionales de promover políticas públicas que busquen la "igualdad" entre los géneros. La lógica subyacente en muchos de los programas estatales dirigidos a las mujeres rurales e indígenas es que el proceso de desarrollo avanzaría mucho mejor si las mujeres estuvieran plenamente incorporadas a él (en lugar de dejar que pierdan su tiempo "improductivamente"). En el caso mexicano, estas políticas se han centrado sobre todo en las mujeres aisladas, promoviendo medidas como el acceso al crédito y al empleo como medios que permitirán a esa mujer integrarse mejor al proceso de desarrollo. 


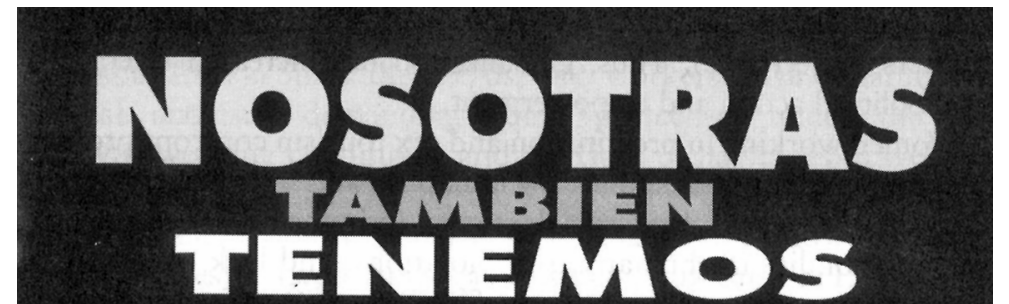

182

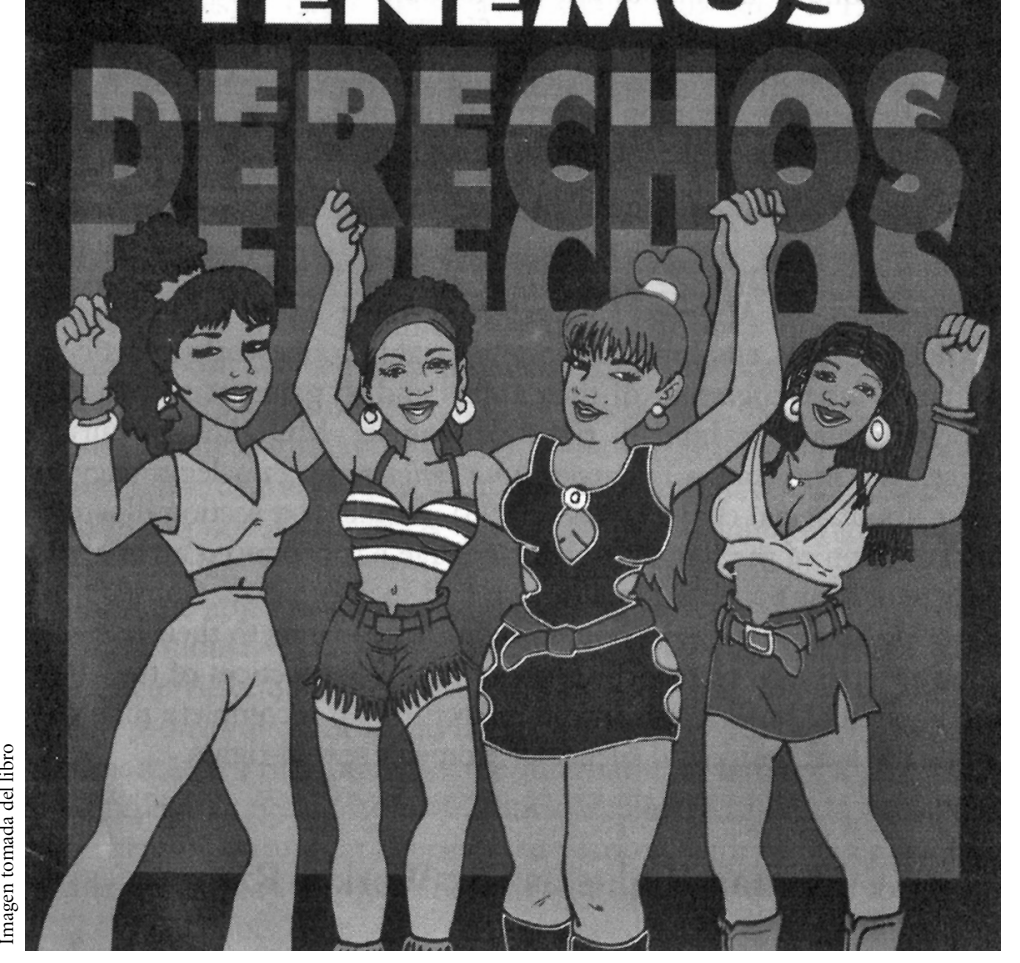

La United States Agency for International Development (USAID) ha sido un importante vehículo para globalizar una agenda de los derechos de las mujeres, que parte del llamado enfoque de "mujer en el desarrollo" (MED), cuya lógica subyacente es que la mujer constituye un recurso no aprovechado susceptible de aportar económicamente al desarrollo. Bajo la influencia de estos discursos globales, otorgar derechos a las mujeres es incorporarlas a un proceso de desarrollo que se ve como el horizonte civilizato- ta que retome y aprenda de estas epistemologías alternativas? En la tercera sección del libro, "Reconceptualizar los derechos", Corinne Kumar, Amalia García Cabezas y Joy Ngozi Ezeilo nos hablan de algunas iniciativas para construir estrategias alternativas de lucha a partir de una concepción de los derechos centrada en las actoras y retomando las experiencias de subalternidad como ventana fundamental para entender la opresión. La feminista de la India, Corinne Kumar, nos comparte en su capítulo "South Wind: Towards a New Political Imaginary" ("Vientos del sur: hacia un nuevo imaginario político") la experiencia de los juicios públicos de mujeres víctimas de violencia. Promotora y coordinadora de estos juicios, que han desempeñado un papel fundamental en la denuncia y, en cierta medida, en la curación espiritual de las mujeres víctimas de violencia, Kumar reflexiona sobre el impacto que puede tener el conocimiento producido en estos espacios. Cuestiona el paradigma de los derechos, junto con el del desarrollo, como vinculados a la globalización corporativa. A partir de esta crítica nos llama a buscar otras historias y otras narrativas para empoderar a las mujeres. Las cortes ofrecen espacios para escuchar otras narrativas, para entender y cuestionar las estructuras de desigualdad. Ellas son las bases para una historia contrahegemónica. Estos vientos del sur traen otras ontologías con nociones diferentes de democracia, poder y gobernanza, otras nociones de igualdad y justicia.

También buscando en las experiencias de la subalternidad las pistas para 
nuevas formas de conocimiento, la feminista cubana Amalia Cabezas nos acerca a las estrategias de resistencia de las prostitutas dominicanas. En su capítulo titulado "Cruces accidentales: turismo, trabajo sexual y derechos de las mujeres en la República Dominicana", hace eco a la propuesta de Kumar al señalar que debemos escuchar las voces silenciadas —en este caso ilegalizadas - de las trabajadoras sexuales, pero, a diferencia de la autora anterior, en vez de rechazar el paradigma de los derechos humanos, llama a expandir su sentido para incluir el derecho a la "agencia sexual". Hablar de los derechos sexuales de las prostitutas permitiría confrontar el discurso normativizante y regulatorio de la ley. Para ella, los discursos de derechos humanos pueden ser un instrumento de empoderamiento para estas mujeres. El trabajo de turismo sexual, como es descrito por las mujeres que lo ejercen, es visto como un reto o un descentramiento a las configuraciones de poder, privilegio, racismo y comodificación sexual. Rechazando convertirse en simples bienes de intercambio comercial, estas mujeres borran las fronteras entre prostitución y romance, trabajo y placer.

El libro cierra con broche de oro con el excelente trabajo de la feminista nigeriana Joy Ngozy Ezeilo: "Feminismo y derechos humanos en los cruces de caminos de África: reconciliar el universalismo y el relativismo cultural", en el que debate con Kumar y plantea que ni el universalismo ni el relativismo han sido instrumentos efectivos para defender los derechos de las mujeres. A partir de su profundo conocimiento del debate en torno a las dicotomías tradición/opresión, derechos universales/liberación, la autora señala que el énfasis en las "prácticas culturales" a favor o en contra opaca la importancia que han tenido el colonialismo, el neocolonialismo y la globalización corporativa como las principales causas de las violaciones a los derechos humanos de las mujeres en África. Plantea que los derechos humanos deben abarcar los espacios privados de la gente y de las corporaciones. Estas reflexiones la llevan a reivindicar la defensa de los derechos humanos como una herramienta a favor de las mujeres. Su lucha como activista en el ámbito de los derechos la llevan a legitimar este espacio tan cuestionado por su colega Corinne Kumar en el capítulo anterior.

Dialogue and Difference: Feminisms Challenge Globalization remueve la conciencia feminista y nos lleva a buscar nuevas alternativas de lucha, a abrir nuestros oídos y nuestro corazón a otras voces y experiencias que nos hablan desde los márgenes. Nos recuerda que el reconocimiento de las diferencias es fundamental en una política feminista que se base en el respeto y la solidaridad. Quisiera cerrar esta reseña con una cita que, me parece, resume la propuesta analítica y política que recorre este libro. Las palabras de Chandra Mohanty, editora de la serie Comparative Feminist Studies, en la que se publica esta antología, nos alientan a recuperar el optimismo de que es posible hacer de nuestras debilidades fortalezas:

"En otras palabras, esta discusión me permite volver a enfatizar la forma en que las diferencias no son nunca simplemente 'diferencias'. Al conocer las diferencias y particularidades, podemos ver mejor las conexiones y elementos comunes, porque no existe frontera o límite que sea total o que nos determine de forma rígida. El obstáculo a superar es ver cómo las diferencias nos permiten explicar las conexiones y los cruces de fronteras mejor y con más precisión, así como especificar la diferencia nos permite teorizar los problemas universales más integralmente. Es esta jugada intelectual la que despierta mi interés porque las mujeres de distintas comunidades e identidades construyan coaliciones y solidaridades más allá de las fronteras" (Chandra Mohanty). 